\title{
Análise das condições de acessibilidade urbana das guias rebaixadas para pedestres em área central de Curitiba- Paraná
}

\author{
Analysis of the urban accessibility of access ramps in the downtown área of Curitiba- \\ Parana city
}

Analisis de las condiciones de accesibilidad urbana de guías rebajadas. en el área central de Curitiba - Parana

\section{Pillar Muzillo}

Mestranda em Engenharia de Construção Civil, UFPR, Brasil

pi.muzillo@gmail.com

Cristina de Araújo Lima

Professora Doutora, UFPR, Brasil cristinadearaujolima@gmail.com 




pelo desenho acessível. Além disso, o conhecimento técnico necessário à implantação do DU é ainda insuficiente no Brasil, dificultando ainda mais sua adoção no país (MINISTÉRIO DAS CIDADES, 2005). A segunda forma, mais comumente utilizada no Brasil, o chamado "desenho acessível", propõe a criação de condições de acesso para pessoas com mobilidade reduzida ou deficiência por meio da instalação de elementos de uso específico, como elevadores, plataformas de acesso e guias rebaixadas para pedestres (MINISTÉRIO DAS CIDADES, 2005). Criadas para auxiliar a transposição de mudanças de nível nas transições de uma calçada a outra, as guias rebaixadas devem apresentar condições mínimas de usabilidade, como regularidade, textura, declividade e manutenção, caso contrário favorecendo tropeços, escorregões ou a trepidação, no caso de cadeiras de rodas (MINISTÉRIO DAS CIDADES, 2005) e carrinhos de bebê. As exigências da NBR 9050/2015 com relação a tais elementos são semelhantes àquelas feitas para o passeio - faixa da calçada por onde transitam os pedestres - devendo ter superfícies firmes, estáveis e antiderrapantes sob qualquer condição, além de não provocar trepidações ou efeitos visuais que causem insegurança no usuário. Além disso, é importante que sejam sinalizadas com a pintura do símbolo internacional de acesso, que indica sua utilização por pessoas em cadeiras de rodas, e com a instalação do piso tátil, para indicar sua presença para pessoas com deficiência visual (ABNT, 2015). A qualidade do piso, por sua vez, deve ser 



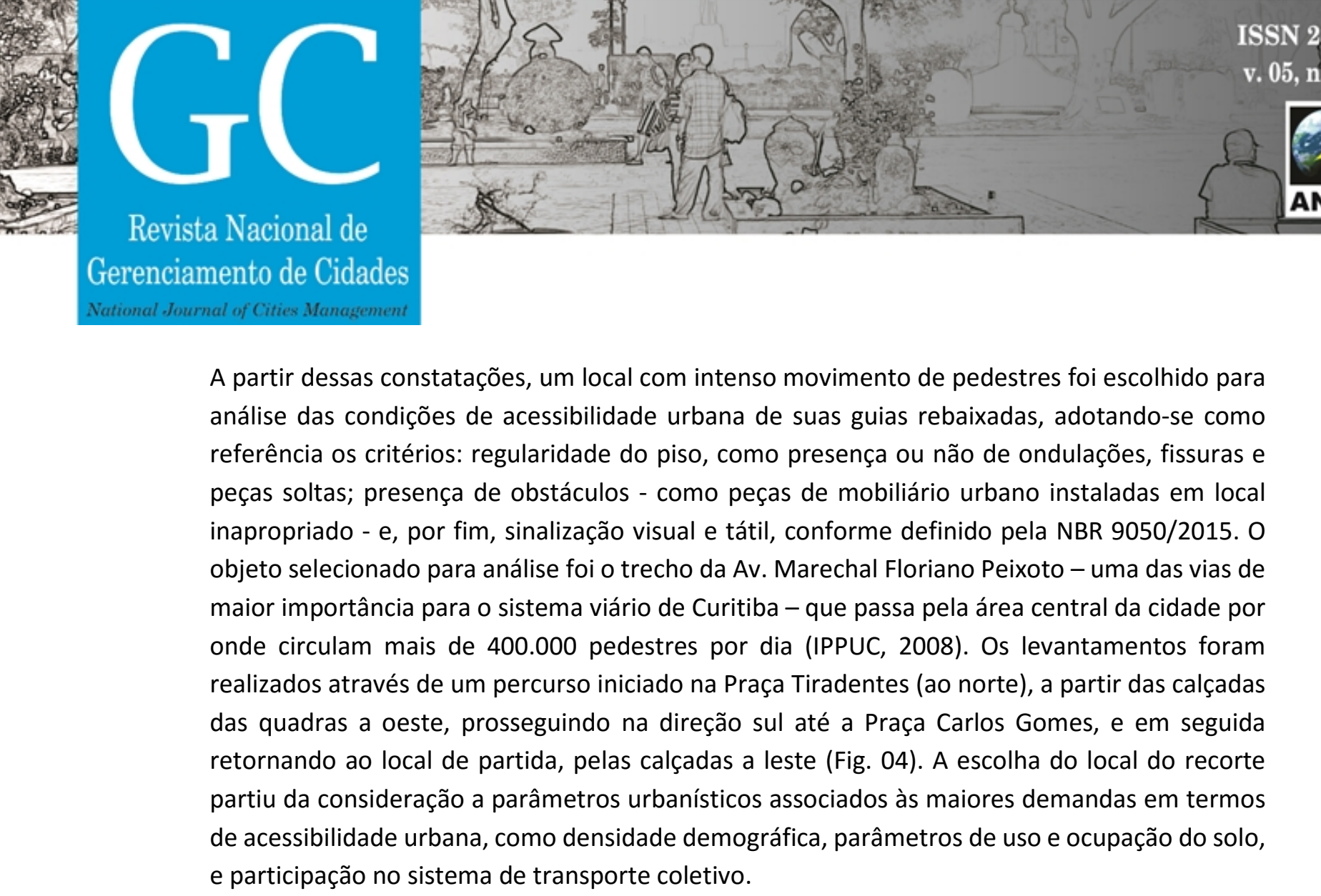

A partir dessas constatações, um local com intenso movimento de pedestres foi escolhido para referência os critérios: regularidade do piso, como presença ou não de ondulações, fissuras e maior importância para o sistema viário de Curitiba - que passa pela área central da cidade por foram realizados através de um percurso iniciado na Praça Tiradentes (ao norte), a partir das calçadas retornando ao local de partida, pelas calçadas a leste (Fig. 04). A escolha do local do recorte partiu da consideração a parâmetros urbanísticos associados às maiores demandas em termos e participação no sistema de transporte coletivo. 

e obstruções que pudessem prejudicar o acesso do pedestre à calçada.

Fonte: AUTORAS (2017)

Pode se observar a presença de ondulações em todas as guias observadas, ainda que sua intensidade variasse de uma guia para outra. Os casos mais graves ocorriam combinados às fissuras, presentes em $68 \%$ das guias no trecho de ida, e $63 \%$ no trecho de volta. Estas, de mesmo modo, apareceram com diferentes profundidades e extensões conforme a guia estudada, variando de marcas superficiais a rachaduras que, nas guias com as condições menos satisfatórias, fragmentavam e afundavam a estrutura (Fig.06). 



CURITIBA. Lei Ordinária no. 11.266/2004: "Dispõe sobre a adequação do Plano Diretor de Curitiba ao Estatuto da Cidade - Lei Federal no 10.257/01, para orientação e controle do desenvolvimento integrado do Município". Disponível em: <https://www.cmc.pr.gov.br/pd_plano_diretor.php>. Acesso em: 21.jun.2017.

DEMPSEY, N. BROWN, C. BRAMLEY, G. The key to sustainable urban development in UK cities? The influence of density in social sustainability. In: progress in planning, no. 77, p.89-141. Elsevier, 2012. Disponível em: < http://www.sciencedirect.com/science/article/pii/S0305900612000189 >. Acesso em: 18.dez.2014.

DISCHINGER, M. JACKSON FILHO, J. M. Can tactile tiles create accessible urban spaces?. In: Space and culture. №. 15, V. 3. p.210-223. Sagepub, 2012. Disponível em: <http://sac.sagepub.com/content/15/3/210>. Acesso em: 24.set.2014.

DUARTE, C. R., COHEN, R. Proposta de metodologia de avaliação da acessibilidade aos espaços de ensino fundamental. In: Anais NUTAU 2006: Demandas Sociais, Inovações Tecnológicas e a Cidade. São Paulo, USP: 2006. Disponível

em: <http://www.proacesso.fau.ufrj.br/artigos/Acessibilidade\%20em\%20Escolas\%20NUTAU\%202006.pdf>. Acesso em: 21.jun.2017.

GEHL, J. La humanización del espacio urbano. 5a ed. Barcelona, Editorial Reverté S.A, 2009.

GRANT, B. Getting to great places: how better urban design can strengthen San Jose's future. In: Spur report. San jose, 2013. Disponível em: <http://www.spur.org/publications/spur-report/2013-12-12/getting-great-places>. Acesso em: 20.abr.2016

HALDEN, D. The use and abuse of accessibility measures in UK passenger transport planning. Disponível em: <http://dhc1.co.uk/content/useandabuseonline.pdf>. Acesso em: 10.mai.2016.

HALDEN, D., JONES, P. e WIXEY, S. Accessibility Analysis Literature Review. Working Paper 3. In: Measuring Accessibility as Experienced by Different Socially Disadvantaged Groups. (atualizado em 2005). Disponível em: <http://www.recordstudy.org/images/record/upload/publications/RESP_mobility_and_health.pdf>. Acesso em: 16.fev.2016.

HANDY, S. L., BOARNET, M. G., EWIG, R., KILLINGSWORTH, R. E. How the built environment affects physical activity: views from urban planning. In: American Journal of Preventive Medicine. N. 23, vol. 2. p. 64 - 73. Elsevier Science, 2002. Disponível em: <http://www.ajpmonline.org/article/S0749-3797(02)00475-0/pdf>. Acesso em: 21.jun.2017.

IMRIE, R. Universalism, Universal Design and equitable Access to the built environment. In: Disability \& Rehabilitation, n. 여, vol.10, Informa UK, 2012. Disponível em: < 10.3109/09638288.2011.624250 >. Acesso em 29.fev.2015.

INSTITUTO DE PESQUISA E PLANEJAMENTO URBANO DE CURITIBA - IPPUC. Bairros mais densos de Curitiba. In: Análise do Censo. Disponível em: 
<http://ippuc.org.br/visualizar.php?doc=http://admsite2013.ippuc.org.br/arquivos/documentos/D356/D356_003_ BR.pdf>. Acesso em: 31.mai.2017.

Plano de Mobilidade Urbana e Transporte Integrado. PlanMob Curitiba. Anexo I-Diagnóstico de acessibilidade. Curitiba, 2008. Disponível em: <http://www.ippuc.org.br/mostrarpagina.php?pagina=35\&> Acesso em: 30.mai.2016.

Instituto Brasileiro de Geografia e Estatística - IBGE. Censo demográfico 2010: Características urbanas do entorno dos municípios. Rio de Janeiro, 2012 . Disponível em: <http://biblioteca.ibge.gov.br/visualizacao/periodicos/96/cd_2010_entorno_domicilios.pdf>. Acesso em: 05.ago.2015.

Cidades. Disponível em: <http://www.cidades.ibge.gov.br>. Acesso em 28.mai.2017.

Kenyon, S., Lyons, G. and Rafferty, J. (2002) Transport and social exclusion: Investigating the possibility of promoting inclusion through virtual mobility. In: Journal of Transport Geography. N. 10 (3). pp. 207-219. Disponível em: <http://dx.doi.org/10.1016/S0966-6923(02)00012-1>.Acesso em 19.set.2017.

LARRAÑAGA, A. M. Análise do padrão comportamental de pedestres. Dissertação de mestrado. Programa de pósgraduação em Engenharia de Produção da UFRS. Porto Alegre, 2008. Disponível em: < https://www.lume.ufrgs.br/bitstream/handle/10183/15294/000677736.pdf?sequence=1 >. Acesso em: 19.set.2017.

LAVERY, I. DAVEY, S. WOODSIDE, A. EWART, K. The vital role of street design and management in reducing barriers to older peoples' mobility. In: Landscape and Urban Planning. vol.35, №. 2 e 3. P. 181-192. Elsevier, 1996. Disponível em: <http://www.sciencedirect.com/science/article/pii/016920469600309X>. Acesso em: 25.abr.2015.

LEI FEDERAL №. 9503/97. Institui o Código de Trânsito Brasileiro. Disponível em: <http://www.planalto.gov.br/ccivil_03/leis/L9503.htm>. Acesso em: 11.jul.2017.

LITMAN, T. Evaluating accessibility for transportation planning measuring people's ability to reach desired goods and activities. Victoria Transport Policy Institute, 2016.

MACE, R. $\quad$ L. $\quad$ Ronald $\quad$ L. Mace. <http://www.universaldesign.com/index.php?option=com_content\&view=article\&id=88:rona Id-I-macefaia\&catid=2196: universal-design\&Itemid=2931>. Acesso em: 06.out.2014

MACHADO, M. H., LIMA, J.P. Avaliação multicritério da acessibilidade de pessoas com mobilidade reduzida: um estudona região central de Itajubá (MG). In: Urbe: Revista Brasileira de Gestão Urbana. V. 07, no. 03. Set/dez. 2015. Disponível em: <http://www.scielo.br/pdf/urbe/v7n3/2175-3369-urbe-2175-3369007003A008.pdf >. Acesso em 30.mai.2016

MINISTÉRIO DAS CIDADES. Implementação do Decreto no..296/04 para a construção da cidade acessível. In: Programa brasileiro de acessibilidade urbana - Brasil acessível. Caderno 3. Disponível em: < http://www.faders.rs.gov.br/uploads/1310575361BrasilAcessivelCaderno03.pdf >. Acesso em: 22.set.2015. 
MOBILIZE.ORG. Divisão modal (\%) cidades. Disponível em: <http://www.mobilize.org.br/midias/estatisticas/divisaode-modais-por-cidades-ii.png>. Acesso em: 13.out.2015

MUZILLO, P. Condições de acessibilidade urbana em passeios: análise em recorte da área central de CuritibaParaná. Dissertação de Mestrado em Engenharia de Construção Civil. Universidade Federal do Paraná, Curitiba, 2016.

NEWMAN, P.W.G. Cities and automobile dependence: a sourcebook. Gower, Aldershot In: COOKE, S. BEHRENS, R. A comparative analysis of the relationship between public transport and land use characteristics. In: Proceedings of the 33rd Southern African Transport Conference (SATC, 2014). Pretoria, CE Projects, 2014. Disponível: <http://hdl.handle.net/2263/45522 >. Acesso em: 21.jun.2017.

PAVANELLI, G., CAVALCANTE, M. M., ROCHA, A. L. da. A pessoa com deficiência no mercado de trabalho formal. In: VII Senabraille.27 a 30 de novembro de 2011.

ROBSON, C. Real world research: a resource for social scientists and practitioner-researchers. 2 ed. Malden: Blackwell Publishers, 2002.

SASSAKI, R. K. Inclusão: acessibilidade no lazer, trabalho e educação. In: Revista Nacional de Reabilitação (Reação). São Paulo, Ano XII, mar./abr. 2009, p. 10-16.

SECRETARIA DO GOVERNO MUNICIPAL. Relatório Acessibilidade - protocolos. Curitiba, 2015. Documento obtido por e-mail.

URBANIZAÇÃO DE CURITIBA S/A (URBS). Itinerários. Disponível em: <https://www.urbs.curitiba.pr.gov.br/transporte/itinerarios>. Acesso em 21.jun.2017.

: O sistema de transporte coletivo de Curitiba - RIT - Rede Integrada de Transporte. In: VIII Ciclo de palestras:

Curitiba, a experiência em gestão urbana. Apresentação. Disponível em: <http://www.imap.curitiba.pr.gov.br/wpcontent/uploads/2014/PDF/14_0111_TRANSPORTE\%20COLETIVO\%20\%20 14.11.2014.pdf $>$. Acesso em 14.out.2015.

YIN, R. Estudo de caso: planejamento e métodos. 2 ed. Bookman, Porto Alegre, 2010.3 